\title{
Los procesos de socialización de docentes de inglés del Programa Nacional de Inglés para educación básica en las primarias públicas de Baja California
}

Márquez-Palazuelos, Ma. del Carmen Enriqueta; Lynn Villezcas, Vicki; González-Medina, Saúl; FongFlores, Jesús Eduardo; Wall-Medrano, Claudia María

Los procesos de socialización de docentes de inglés del Programa Nacional de Inglés para educación básica en las primarias públicas de Baja California

Revista Educación, vol. 42, núm. 2, 2018

Universidad de Costa Rica, Costa Rica

Disponible en: http://www.redalyc.org/articulo.oa?id=44055139003

DOI: https://doi.org/10.15517/revedu.v42i2.23442

Esta obra está bajo una Licencia Creative Commons Atribución-NoComercial-SinDerivar 3.0 Internacional. 


\title{
Los procesos de socialización de docentes de inglés del Programa Nacional de Inglés para educación básica en las primarias públicas de Baja California
}

\author{
The Socialization Process of EFL Teachers Working in Baja California with the National English Program for Basic \\ Education
}

Ma. del Carmen Enriqueta Márquez-Palazuelos [1]

Universidad Autónoma de Baja California, México

DOI: https://doi.org/10.15517/revedu.v42i2.23442

carmenm@uabc.edu.mx

Vicki Lynn Villezcas [2]

Universidad Autónoma de Baja California, México

vvillezcas@uabc.edu.mx

Recepción: 22 Febrero 2016

Saúl González-Medina [3]

Universidad Autónoma de Baja California, México

salgoens@uabc.edu.mx

\section{NotAS DE AUTOR}

[1] Profesora investigadora de la Facultad de Idiomas de la Universidad Autónoma de Baja California. Cuenta con Licenciatura en Enseñanza del Inglés por la Normal Estatal de B.C., Maestría en Investigación Educativa por el Instituto de Investigación Educativa de la UABC y actualmente es candidata a Doctorado en Educación en la Universidad Tecnológica de Tijuana, B.C. Es miembro desde el 2009 del Programa para el desarrollo profesional docente (PRODEP) antes (PROMEP), que otorga la Secretaría de Educación Pública a nivel nacional. Colabora en la formación de docentes en el Programa de Licenciatura de la Facultad de Idiomas desde 2006. Los cargos que ha desarrollado son: coordinadora del Centro de Idiomas, coordinadora de la Maestría en Docencia y Coordinadora del area de Investigación y Posgrado de la Facultad de Idiomas de la UABC. Ha dirigido 8 tesis de maestría y participado en congresos tanto en México como en el extranjero con diversas ponencias. Ha participado en 2 proyectos de investigación a nivel nacional "La investigación en el área de enseñanza y aprendizaje de lenguas" y "La enseñanza del inglés en primarias públicas de México en colaboración con otras universidades del país. Sus áreas de interés son la evaluación, la formación docente, las creencias y las inteligencias múltiples. Así mismo ha publicado 2 capítulos de libro y10 artículos. Miembro del Comité Editorial de Mextesol 2008-2014 y colaborado en el arbitraje de artículos para la Universidad de Sonora y el Consejo Mexicano de Investigación Educativa, A.C. (COMIE).

[2] Profesora de Asignatura en las Licenciaturas de Docencia y Traducción de la Facultad de Idiomas. Obtuvo su Maestría en Educación (MA) de San Diego State University (SDSU), Estados Unidos y Licenciatura en Ciencias de la Educación, también de San Diego State University. Tiene estudios de especialidad en Educación Bilingüe de la misma institución, Diplomado en Lingüística Aplicada de la Universidad Nacional Autónoma de México. Sus áreas de interés en investigación son: formación docente, literacidad, pragmática, estrategias de enseñanza-aprendizaje, evaluación de competencia lingüística y educación en línea. Se ha desempeñado tanto como coordinadora de maestría en docencia así como, profesora de grado y posgrado en los cursos de principios de investigación y seminario de investigación, entre otros. También se ha desempeñado como directora de tesis y sinodal en exámenes profesionales de futuro personal docente.

[3] Obtuve mi doctorado en investigación educativa en el año 2013 en la Escuela Normal Superior de Ciudad Madero A.C., tengo una maestría en docencia por la Universidad Autónoma de Baja California (UABC) y un B.Phil.Ed. en enseñanza de inglés por la Universidad de Exeter. Actualmente me desempeño como docente, de tiempo completo, en la Facultad de Idiomas de la UABC en la cual, durante los últimos cinco años, he impartido cursos en los niveles de licenciatura y posgrado de las áreas de didáctica, formación docente, investigación y diseño de planes de estudio; así también, he sido director de tres tesis de maestría y sinodal en 8 tesis de maestría en el programa de maestría de Docencia de la misma Facultad. Actualmente, participo como coinvestigador en el proyecto de investigación "Revealing the educational experiences and needs of Los Otros DREAMers", otros proyectos en los que he participado recientemente son "Diagnóstico de la enseñanza de Inglés en las primarias públicas del Estado de Baja California" y "La percepción académica de los estudiantes de Licenciatura de Idiomas y Traducción". En este momento ocupo el puesto de responsable de las áreas de vinculación, intercambio estudiantil y educación continua de la Facultad. Otros puestos desempeñados recientemente son coordinador del área de formación profesional y vinculación y coordinador de la carrera de traducción nivel licenciatura.

[4] Profesor de tiempo completo de la Universidad Autónoma de Baja California, posee el grado de Maestro en Lenguas Modernas y actualmente cursa el segundo año de doctorado en la Universidad Nacional de Estudios a Distancia en Madrid, España. Las líneas de investigación en la cual se centra son la lingüística aplicada y la formación de profesorsdo en lenguas extranjeras, en particular, la enseñanza del inglés y francés. Actualmente, participa en proyectos orientados a la formación de docentes en el sector público y privado en busca de favorecer la formación inicial de estudiantes de la Facultad. También, se desempeña como coordinador académico de la Facultad de Idiomas extensión Ensenada y como responsable de estudiantado egresado y titulación.

[5] Ha trabajado para la Universidad Autónoma de Baja California en la Facultad de C. Marinas, Idiomas, y Medicina en el área de inglés. Es candidata al doctorado por el Instituto Mc Laren de Pedagogía Critica. Cuenta con 32 años de experiencia. Entre sus intereses están ingles con propósitos específicos, la competencia plurilingüe e interculturalidad así como PNIEB. Ha sido tallerista del área de vinculación de la Facultad de Idiomas desde el 2007 y ha representado a la UABC en congresos estatales y nacionales con talleres. Entre ellos se encuentra su participación en la Convención Internacional de Mextesol en el taller "A Framework for assesment in a competency-based program" en el 2010. 
Jesús Eduardo Fong-Flores [4]

Universidad Autónoma de Baja California, México

jfong@uabc.edu.mx

Claudia Maria Wall-Medrano [5]

Universidad Autónoma de Baja California, México

claudiamaria@uabc.edu.mx

\section{Resumen:}

El presente estudio busca conocer los procesos de socialización del personal docente que labora en el Programa Nacional de Inglés a nivel primaria y preescolar en Ensenada Baja California, México, para identificar, por un lado, la construcción de la identidad docente desde un enfoque socio pedagógico y; por otro, dar cuenta del contexto en el cual se encuentra inmerso para así contribuir al diálogo en apoyo a acciones que mejoren su entorno. Nuestro propósito no es solo generalizar sobre la construcción de identidades, sino también profundizar sobre el proceso que experimentan docentes que trabajan en el programa. Para enmarcar esta información se realizó un estudio cualitativo con orientación a estudio de caso. Los datos fueron recolectados a través de entrevistas semiestructuradas a un grupo de sujetos clave utilizando el método de análisis por categorías para organizar y concretar los resultados. La intervención circunscribe dos tipos de docentes; aquel grupo que cuenta con una formación pedagógica base, producto de su paso por programas formales de instrucción a nivel de universidad y, otros grupos que obtuvieron su trabajo como docentes por el manejo del inglés o su pertenencia al sistema educativo. También es significativa la manifestación de una falta de inducción al programa para su ingreso al contexto escolar, la precariedad de las condiciones laborales, la falta de apoyo para la capacitación pedagógica y el poco reconocimiento de la imagen del personal docente de lenguas por la sociedad.

Palabras ClaVE: Socialización docente, formación docente, cultura organizacional, identidad profesional, proyección docente.

\section{Abstract:}

The present study attempts to create a better understanding of the socialization process, which teachers working in the National English Program for Basic education in Ensenada Baja California, Mexico, must experience. On one hand, findings are based on a study of the construction of teachers' identities from a socio-pedagogical perspective and on the other hand. on a review of the context in which they are immersed. Our purpose is not only to generalize results as to achieve a deeper understanding of the process which teachers working in the program experience, and clarify how these results influence the construction of their own identities contributing to dialog which support actions to improve their context. Descriptive data was collected through semi-structured interviews with a group of key subjects using categorization as a method of analysis to identify results. It is worth mentioning that within the findings, two classes of teachers were typified: those who command basic pedagogical knowledge because they have gone through formal training and those who have no pedagogical training, but were recruited for the job because they speak English or belong somehow to the educational system. It is also notable to mention the absence of an induction into the program upon initiating activities in an educational setting, the precarity of working conditions, the minimal support for further pedagogical training and the disesteem of communities in regards to language teachers' social status.

KEYWORDS: teachers' socialization, teacher training, organizational culture, professional identity, teachers' social projection.

\section{INTRODUCCIÓN}

La socialización docente ha sido investigada principalmente en relación con los procesos de formación inicial y su incorporación a la profesión docente. Estos procesos de socialización confluyen en el individuo para considerarse profesional de la enseñanza. Por tanto, este estudio busca identificar descriptores de su inserción en el campo laboral, la percepción que tienen del programa, su propia labor docente y la identidad profesional que han desarrollado a la fecha; en resumen, detallar su proceso de socialización.

Aclaramos que se procura enmarcar, específicamente, el proceso que siguen docentes de inglés en la población de Ensenada, Baja California, México, al incorporarse al contexto de trabajo del Programa Nacional de Inglés independientemente de si son personas recién egresadas de algún programa de formación docente o si ya cuentan con algunos años de experiencia como docentes de lenguas. Nos enfocamos en estos grupos docentes de inglés por representar una profesión vulnerable a las cambiantes políticas lingüísticas del 
país en el panorama de educación básica como parte del plan educativo, tras la institución relativamente reciente del Plan Nacional de Inglés en educación básica en el 2009.

\section{Antecedentes}

Con base en la Reforma Educativa planteada en México a partir del Plan de Desarrollo 2007-2012 y los objetivos señalados en el Programa Nacional de Educación 2007-2012 (H. Cámara de Diputados, LX Legislatura (2007), la Secretaría de Educación Pública dio inicio en 2009 a un Programa Nacional para la enseñanza del Inglés en Educación Básica, denominado PNIEB, con el objetivo de que el estudiantado, en todos los niveles de educación básica (preescolar, primaria y secundaria), obtengan los conocimientos para participar en prácticas sociales orales y escritas que le proporcionarán la capacidad de interaccionar adecuadamente en un contexto diferente a su lengua materna, específicamente el inglés.

Destacamos que, previo a la implementación del PNIEB existían en 21 Estados de la República Mexicana programas estatales de Inglés en primarias públicas sin que hubiera homogeneidad entre ellos (Ramírez, et. al., 2012). Precisamente, con la implementación del Programa Nacional se pretendía unificar criterios para asegurar resultados más exitosos. Este proyecto era bastante ambicioso y requería de grandes insumos de dinero y de recursos humanos que no pudieron ser alcanzados. Al final del sexenio 2006-2012, el programa quedó suspendido por un tiempo en algunos estados del país (Calderón, 2015).

En el siguiente régimen se reactivó el programa al trasladarse a los gobiernos estatales, que serían responsables de su desarrollo, y se dio a conocer como PIEB. En el Estado de Baja California el programa se instituyó únicamente en las escuelas públicas de tiempo completo y se inició con docentes de preescolar y primaria que tuviesen conocimiento del inglés y que formaban parte del sistema educativo (Subsecretaria de Educación Básica, Sistema Educativo Estatal, 2015).

A partir del 2015, el nuevo régimen federal presentó otra reforma educativa, que incluía una propuesta reajustada a la anterior para la implementación del inglés a nivel educación básica. Esta se dio a conocer como el Proyecto Nacional de Inglés (PRONI). La propuesta, esta vez lanzada a través del sistema educativo estatal, fue impulsar estratégicamente el aprendizaje del idioma inglés en los planteles escolares ahora desde una perspectiva transversal, dentro y fuera del aula. La implementación de este Programa Nacional de Inglés (PRONI) inicia en 2016 y comprende el periodo 2015-2019 (Subsecretaria de Educación Básica, 2016).

Durante el sexenio 2006-2012 e inicios del sexenio 2013-2019, los principales protagonistas del Programa de Inglés en Ensenada, Baja California han sido las mismas personas. Es decir, se cuenta con la misma coordinación, las mismas escuelas de tiempo completo y el mismo personal docente participantes en la asignatura de inglés. Las condiciones laborales y procesos de socialización son similares, en el sentido de que cuentan con los mismos factores característicos como son: entorno institucional, alumnado y docentes (Gobierno del Estado de Baja California, 2016).

El programa, sin embargo, sí ha cambiado y es importante conocer a docentes que permanecen desde el antes y hasta el después de los cambios e identificar sus necesidades para ser atendidas. Desde la perspectiva de Díaz Barriga e Inclán (2001), un componente trascendental para el éxito de las reformas educativas es aquel que directamente implementa los programas en el aula: el personal docente. Por tal motivo, consideramos imperante cuestionarnos: ¿Quiénes constituyen el personal docente que colabora con el Programa Nacional de Inglés PNIEB-PRONI? ¿Cuál es su perfil? ¿Cómo son los procesos de socialización a los que se han expuesto? ¿Cómo estos procesos determinan su identidad como profesionales?

El presente estudio buscó responder a estos interrogantes al presentar un acercamiento a docentes que incursionan en el programa, considerando como eje esencial los procesos de socialización a los que se han expuesto, y las implicaciones que este hecho revela. 


\section{Revisión de la bibliografía}

La socialización docente. Existen varias posturas en relación con la socialización de profesorado. Al realizar una revisión de la bibliografía referente al tema se presenta una gran diversidad de trabajos que sitúan este proceso con aproximaciones distintas. Estas enriquecen su estudio y dan mayor enfoque a una temática muy compleja.

De inicio, Benegas (2002) indica que se puede analizar el tema desde un sentido estricto o de un sentido amplio. Ciertamente, la naturalidad de la formación docente representa una postura que ubica a profesionales de las lenguas como personas preparadas y capaces de hacer frente a su práctica profesional. En este sentido, Davini (1995) plantea que el sentido estricto examina la biografía escolar de futuro personal docente, su preparación inicial o de grado y su socialización profesional. Por otro lado, el sentido amplio explora conocimientos, habilidades, costumbres, rutinas que conforman un proceso de aprendizaje de la enseñanza, un aprender a enseñar, como proceso de socialización que no está ajeno a las influencias previas a la formación, en la formación inicial, y en el contexto del trabajo profesional (González, 1995).

Imbernón (2007), complementa lo anterior con una afirmación más ubicada en definiciones terminológicas, al mencionar que el término "formación" hace alusión a una capacitación o preparación formal, mientras que el término "socialización" hace referencia al momento "en el que el nuevo profesor se integra como miembro activo y participante en el colectivo profesional” (p.58). Al proceso de formación se agregan las creencias previas que trae cada docente y que influyen directamente en los conocimientos que adquiere y en el proceso de socialización en la práctica (Latorre y Blanco, 2007).

En cambio, Ramírez et al. (2012) perciben la socialización docente como un fenómeno que se presenta en cinco rubros problemáticos y agregan temas de consideración contextualizadas al proceso: 1) Su interacción con las políticas educativas (estos casos comprenden, entre otros, el conocimiento, aceptación y congruencia entre discursos y práctica de programas para la enseñanza del ingles); 2) Su acercamiento a los planes de estudio (existencia, asignación de horas, status, participación de las editoriales); 3 ) Su identidad, formación y condiciones laborales; 4) Su metodlogia y prácticas docentes y su actualización y 5) Los recursos educativos que tiene a su alcance.

Zeichner y Gore (1990), en contraste, sitúan la incursión del profesorado en el medio profesional desde un ámbito más sociológico. En su artículo presentan las influencias al proceso de socialización profesional desde cuatro diferentes perspectivas: a) La etapa previa al entrenamiento, b) La formación inicial, c) La experiencia en el campo y d) La inducción. Coinciden con Benegas (2002) y Day (2005), en ubicar el inicio del proceso de socialización antes de incursionar en su formación profesional. Agregan que la socialización del profesorado es un proceso complejo donde una persona incursiona en la profesión de la docencia y llega a ser integrante de una comunidad de docentes, donde adquiere valores, habilidades, conocimientos y experiencias acumuladas en el tiempo.

Destacamos que este estudio adopta la concepción propuesta por Zeichner y Gore (1990) como punto de partida. Esto, por considerarla la más concretada para visualizar los indicadores en la socialización docente en general, y en el personal docente del idioma inglés de educación básica en particular. Además, conlleva al conocimiento de los procesos de formación y proyección docente, así como a descubrir su identidad dentro y fuera de la cultura organizacional desde una perspectiva sociológica, que característicamente orienta fenómenos que se presentan en el contexto escolar.

A continuación, se procura profundizar en los rubros mencionados por Zeichner y Gore (1990), enmarcando la información con descriptores más incluyentes, con el fin generar una visión global. Para ello, la etapa previa al entrenamiento, la formación inicial y la formación continua se han caracterizado como formación docente, la experiencia en el campo y proyección docente como elemento integrador de su identidad profesional, y su inducción e incorporación al contexto escolar como parte de la cultura organizacional. 
La formación docente. El acercamiento o etapa previa al entrenamiento, así como la formación inicial y continua docente, sobre todo para los profesores y profesoras de inglés del Programa Nacional de Inglés en México, son elementos importantes y complejos de un proceso de socialización que requiere una atención contextualizada. Estos elementos se definen a partir de parámetros que buscan condicionar su formación, tanto inicial como continua, destacando influencias previas y competencias con las cuales debe contar. En este marco también existen políticas que han determinado las características y aptitudes que deberían tener.

Bazdresch (2006) hace un notable señalamiento al afirmar que la formación docente debe dimensionar la práctica educativa como objeto de conocimiento en sus dimensiones política, escolar y áulica (formación inicial). Este autor sugiere que se incluya la reflexión sobre la práctica docente a partir de una experiencia empírica, de los retos que surgen en la práctica y la integración de la teoría existente, para formular nuevos conocimientos.

Con base en las afirmaciones anteriores, se simplifica el proceso de formación docente con dos ejes esenciales: la formación inicial y la formación continua, en donde se busca que el personal docente sepa integrar los conocimientos y habilidades, así como una variedad de capacidades que permitan un desarrollo efectivo en el campo educativo. El ejercicio de enseñar demanda que durante la etapa previa a su incorporación al campo laboral, los cuerpos docentes sepan comprometerse con su formación y con su desarrollo profesional, tal y como señala Day (2005), quien considera la profesión docente como un desarrollo continuo que, partiendo de una formación inicial, busca una actualización en los conocimientos disciplinares, didácticos o tecnológicos para evolucionar, mejorar y adaptarse, mediante la práctica reflexiva, a la realidad institucional.

La identidad profesional. La construcción de la identidad profesional del personal docente tiene que ver directamente con la socialización del profesorado, de donde surgen varias dimensiones. Se podría decir que los procesos de socialización son el crisol de donde emanan aspectos como la construcción de identidad en la consolidación de prácticas educativas exitosas y, por ende, en su crecimiento profesional.

Varios estudios han contribuido a explicar la identidad desde diferentes perspectivas. Danielewicz (2001) explica la identidad como nuestro entendimiento de quiénes somos y qué pensamos del otro. Norton (1995), por su parte, establece que la identidad se define en cómo el individuo se percibe en relación con el mundo, cómo se construye esa relación a través del tiempo y espacio y cómo el individuo percibe sus posibilidades futuras. Desde una perspectiva lingüista, Van Dijk (1999), fundador del análisis del discurso, define la identidad social agregándolo a la orientación sociocognitiva, como un constructo mental socialmente compartido, por lo cual utiliza esquemas mentales para una acción social, política o cultural.

Norton (1995) y Van Dijk (1999) señalan que la identidad profesional se sujeta a una construcción y reconstrucción continua, empezando desde su formación como docente al cuestionarse: ¿Quién soy?, ¿Quién quiero ser?¿Qué puedo hacer? Se buscan respuestas a través de procesos de reflexión que les conducen al desarrollo de sus competencias profesionales durante su formación, para luego ser contrastadas al integrarse al contexto educativo.

Asimismo, el sociólogo francés Dubar (1991) pone en el centro de la construcción de la identidad los vínculos entre sujetos e instituciones sociales. Para él la identidad es un proceso de interacción complejo que se construye en la interacción con otros seres, en contextos históricos y socialmente estructurados. Esta complejidad la ilustra Márquez (2011) al explicar que, en la nueva realidad, la relación del profesorado de inglés con autoridades, padres y madres de familia, y docentes de grupo, en el contexto del Programa Nacional de Inglés (en este caso refiriéndose a su aparición como PNIEB), se ha manifestado como un área potencial de problema. En su trabajo, una parte del profesorado reportó tener escasa comunicación con los directores y directoras de la institución.

También aclaran Fierro y Martínez (2015) que, en el contexto del Programa Nacional de Inglés, existe una crisis de identidad causada por la heterogeneidad de perfiles académicos que laboran en las instituciones 
educativas, lo que dificulta la conformación de una comunidad de práctica integrada para enfrentarse a los retos de la implementación del Programa y construir una identidad profesional con reconocimiento.

Cultura organizacional. Las instituciones u organizaciones son parte fundamental del funcionamiento de una sociedad. Ellas reflejan la forma de pensar, el tipo de relaciones humanas y la vida social inherente a una comunidad. Las escuelas, como organizaciones educativas, además de reflejar esta realidad, tienen una relevancia primaria al ser los instrumentos a través de los cuales se construyen, se mantienen y se modifican los tres aspectos sociales anteriormente mencionados. Las escuelas son el espacio propicio para el desarrollo de la socialización y el conocimiento que la sociedad requiere (Peteiro, 2007). Por lo tanto, se vuelve vital conocer ampliamente la dinámica social de sus integrantes, como resultado de diversos procesos de investigación.

Según Vaillant (2007), cuando se establece una relación entre la sociedad, el individuo y la cultura profesional se obtiene una red compleja, caracterizada por múltiples variables, las cuales se encuentran en constante movimiento y requieren que se les mantenga en seguimiento continuo. Así mismo, menciona que aunque la cultura profesional es parte de la cultura organizacional de una institución y refleja a la sociedad misma, a la primera se le otorga el sentido particular de profesión, la cual lleva integrados aspectos políticos, ideológicos, culturales y sociales que, en su conjunto, promueven el sentido de colectividad, identidad y pertenencia.

La investigación realizada por Brito, Araneda, et. al. (2005) expone que la integración profesional docente presenta una serie de dificultades de diversa índole que pueden impactar en la autoestima e imagen profesional del sujeto mismo. Dichas dificultades se refieren a aquellas inherentes al campo académico como: su situación laboral, su realidad cultural, su dominio de competencias y sus interacciones con sus pares, y a la organización como el clima organizacional y el escolar.

Considerando este marco, se conceptualiza la escuela como una organización social dentro de la cual se genera la socialización del profesorado mediante la negociación entre la imagen ideal de docente, la visión de las otras personas sobre su trabajo y sus propias percepciones de lo que necesita; proceso a través del cual se construye la identidad (Corley, 1998).

Así, el personal docente que recién ingresa con una asignatura nueva a la institución percibe sus principales puntos guía para referir su proceso de socialización profesional como son: estudiantes, colegas, autoridades y los padres y madres de familia. Las relaciones que logre cimentar con cada uno de estos grupos determinan las posturas, actitudes y creencias que rigen la cultura organizacional; así también, afectan o reconstruyen la autopercepción de su identidad y de su rol (Pérez, 2007).

De las generalizaciones anteriores, es necesario puntualizar que los estudios de la cultura organizacional deben ser observados en contexto. Al tratar de describir, explicar y entender la cultura organizacional de los centros escolares, es conveniente darse cuenta de que cada centro refleja una realidad propia, en donde las formas de interrelación, prácticas de actuación y sistemas de creencias así como valores son únicos (Gairín y Armengol, 2003).

\section{Metodología}

Por su misma naturaleza, esta investigación se centró en el enfoque cualitativo. La investigación cualitativa, según Vasilachis (2006), busca evaluar el proceso de los sucesos sin manipular la información. Más bien se centra en encontrar el significado de las acciones de los sujetos a través de las percepciones e interpretaciones que estos le dan a su propia realidad.

En este proceso, donde el sujeto docente se vuelve el centro del estudio, se buscó definir al personal docente y su desempeño a partir de su trabajo cotidiano en un contexto real específico (Veiravé, Ojeda, et. al., 2006). Su corte permitió generar categorías o códigos que concuerdan con las preguntas de investigación y dan lugar al análisis. 
Así dio inicio la transcripción de entrevistas, donde se fueron identificando unidades de texto que concordaban con las preguntas de investigación. Estos mismos se plasmaron en una matriz que permitió jerarquizar dichos códigos, comparar los datos obtenidos, interpretarlos, e identificar relaciones, atribuyéndoles así un significado (ver tablas 1-5).

Esta secuencia dio lugar al análisis, de donde se originaron las categorías o los códigos propuestos en un principio, que se redefinieron a partir de la revisión bibliográfica-académica (sistema deductivo). A medida que se fueron revisando los datos recogidos, surgieron otras categorías (sistema inductivo) que enriquecieron su interpretación. Combinando ambos procesos se contó con un sistema deductivo-inductivo con el cual se finalizó el análisis de la información (ver tablas 1-5).

De acuerdo con Balderas (2013), este método ha sido utilizado anteriormente para identificar procesos de identidad profesional correspondientes a la docencia, porque permite entender y comprender el contexto cotidiano del personal docente, así como, la significancia que tienen sus experiencias y acciones habituales propias del ámbito escolar en el que se desenvuelve. Así también, las narrativas representan las dinámicas colectivas, las cuales determinan el proceso de socialización del maestro o de la maestra a partir de la interacción entre su identidad, su cultura profesional y la cultura organizacional de la escuela en la que se desempeña (Veiravé et al. 2006).

El método basa su validez y confiabilidad en la credibilidad y coherencia que se obtiene de las diferentes narrativas obtenidas en las entrevistas a partir de la honestidad personal de quienes narran e investigan, y del interés de ambos grupos por llegar a términos comunes que definan conclusiones consensuadas que se acerquen a la verdad (Bolívar y Domingo, 2006). Además, estos mismos autores mencionan que la triangulación de las evidencias se logra al ser diferentes personas investigadoras quienes interpretan las narraciones derivadas de las entrevistas semiestructuradas. Como consecuencia, al utilizar la teoría como un eje que fundamenta la narración y la interpretación, dan la certeza a los resultados que pretenden reflejar la verdad (Saiz, 2014).

Contexto. La investigación se llevó a cabo con docentes pertenecientes al Programa Nacional de Inglés que laboran en primarias de tiempo completo en la Ciudad de Ensenada, Baja California México.

De acuerdo con información proporcionada por la coordinadora del Programa de Ingles, existen en la Ciudad de Ensenada 25 escuelas primarias y 10 preescolares de tiempo completo en 2014, ampliándose estas en 2017-2018 a 174 escuelas, según informo la delegada del Sistema Educativo Estatal (SEE), Lozano Lopez (2017). Sin embargo, no todas cuentan con el Programa de Inglés, ya que es elección de las direcciones si se incorporan o no al Programa. Así, la cifra total presenta 19 docentes de inglés que trabajan en este rubro (Subsecretaría de Educación Básica, Sistema Educativo Estatal, 2017).

Participantes. Para la selección de los sujetos participantes en este estudio, se utilizó una muestra por conveniencia, la cual es un procedimiento de muestreo no probabilístico donde el elemento se autoselecciona o se ha seleccionado debido a su fácil disponibilidad (Kinnear y Taylor, 1998). Los sujetos participantes se seleccionaron al lanzar un llamado a docentes que laboran en escuelas de tiempo completo (ETC), a través de la coordinación del programa de inglés.

Las ETC son escuelas públicas de educación básica creadas en 2008 por el nuevo Plan Nacional de Desarrollo 2013-2018 para implementar un nuevo enfoque en donde se genera una cultura que promueve nuevos esquemas de organización, dinámicas de trabajo, sistema organizacional y procesos de socialización (Urrutia, 2013). Estas mismas extienden la jornada escolar a 8 horas en comparación con las escuelas tradicionales que cuentan con horario de 5 horas diarias, con el fin de propiciar el logro de aprendizajes con calidad en un marco de equidad, así como atender las dificultades y necesidades educativas de todo el alumnado que asiste a ellas.

Ante la incorporación de un nuevo esquema para las escuelas de tiempo completo se cuenta con un plan curricular establecido. Este considera materias como español, matemáticas, historia, geografía, cívica y 
ética, educación física, artística e inglés; sin embargo, esta última aún presenta diversas interrogantes para consolidarla como un programa adecuado a las necesidades actuales (LaPointe, 2011).

$\mathrm{Al}$ responder el profesorado al llamado, se contó con la participación de nueve docentes que laboran en escuelas primarias de tiempo completo en la ciudad de Ensenada, Baja California. Se integraron como participantes ocho mujeres y un hombre en un rango de edades que fluctuó de 23 a 52 años, con distintos perfiles de instrucción.

De este grupo de nueve docentes, cuatro contaban con una Licenciatura en Docencia de Idiomas, uno con Licenciatura en Negocios Internacionales, una con estudios normalistas como educadora y uno con estudios de nivel medio superior, Diplomado en Docencia por San Diego State University y conocimiento del inglés por haber vivido en Estados Unidos.

Instrumento. Para conocer los procesos de socialización del grupo de docentes participantes se llevó a cabo una entrevista semiestructurada. Su ordenación se asentó en 25 preguntas referentes a su vida profesional, sus experiencias de socialización en la institución en la que laboran, su desempeño laboral y su visión a futuro. De las 25 preguntas, 7 se enfocan en la información general del sujeto, 3 en su ingreso a la docencia, 6 en su identidad profesional y 9 en el contexto laboral.

Las entrevistas se apoyaron con la grabación para asegurar la fidelidad de la información. Así también, el proceso se llevó a cabo con un permiso escrito firmado por los sujetos participantes con la autorización del uso de las grabaciones para fines de este estudio y un etiquetado que ayudó a conservar la confidencialidad.

\section{RESUltados Y ANÁLISIS}

Los resultados de las entrevistas a docentes que laboran en el programa de inglés, de manera general, proporcionan un acercamiento a los cuestionamientos postulados a inicios de este estudio, en específico, y delinean influencias al proceso de socialización profesional, en general, al profundizar en el proceso de socialización docente.

Para organizar los resultados, se ha utilizado el modelo en espiral propuesto por Miles y Huberman (1984), conformado por tres etapas (reducción de la información, estructuración, presentación, extracción de conclusiones y verificación) que, partiendo de la recopilación de la información, forman un proceso interactivo y cíclico.

Dicho de otra manera, se procuró convertir un concepto abstracto (la información recabada) en uno empírico, susceptible de ser analizado a través de la extracción de conclusiones y verificación. El proceso tiene su importancia en la posibilidad de evitar errores frecuentes en un estudio de caso, cuando no existe relación entre la respuesta en una entrevista y su conceptualización.

En un acercamiento inicial a los resultados de este estudio se dimensionaron los tres grandes rubros referidos anteriormente que, desde el ámbito sociológico, aportan a la socialización docente. Estas comprenden: primeramente, la formación inicial, que ayuda a entender el proceso que lleva a convertir a estudiantes o profesionistas en docentes; la identidad profesional que nos lleva a conocer, en términos de los saberes específicos de su profesión, su proyección en la identidad; y la cultura organizacional como ámbito del proceso de socialización.

Formación docente. El primer rubro, que alude a la formación docente, incorpora la etapa previa al entrenamiento y la formación inicial identificadas por Zeichner y Gore (1990), y da lugar a preguntarnos, ¿Quiénes se incluyen como docentes en el Programa Nacional de Inglés?, ¿cuál es su perfil? En la revisión de las entrevistas se identificaron descripciones sobre su trayectoria previa, edad y formación inicial de los nueve docentes que participaron en el estudio. A continuación se presentan en la Tabla 1 los resultados obtenidos: 
TABLA 1

Formación docente Programa Nacional de Inglés

\begin{tabular}{|c|c|c|c|}
\hline Docente & Edad & $\begin{array}{l}\text { Etapa previa: } \\
\text { Influencias externas }\end{array}$ & Formación \\
\hline Olga & 52 & $\begin{array}{l}\text { Padres fallecidos } \\
\text { Gusto por el idioma }\end{array}$ & No cuenta con formación inicial. Diplomado. \\
\hline Valeria & 34 & $\begin{array}{l}\text { Administradora } \\
\text { Facilidad por el } \\
\text { idioma }\end{array}$ & $\begin{array}{l}\text { Cuenta con formación inicial en docencia de } \\
\text { idiomas } \\
\text { Diplomado }\end{array}$ \\
\hline María & 26 & $\begin{array}{l}\text { Madre docente la indujo } \\
\text { Trabajó programa piloto }\end{array}$ & $\begin{array}{l}\text { Cuenta con formación inicial en docencia de } \\
\text { idiomas } \\
\text { Diplomado }\end{array}$ \\
\hline Silvia & 23 & $\begin{array}{l}\text { Padres docentes } \\
\text { No quedó en la normal } \\
\text { Gusto por enseñanza de idiomas }\end{array}$ & $\begin{array}{l}\text { Cuenta con formación inicial en docencia de } \\
\text { idiomas } \\
\text { Cursa programa de maestría Diplomado }\end{array}$ \\
\hline Haydeé & 51 & $\begin{array}{l}\text { Padre docente } \\
\text { Repatriada } \\
\text { Sin trabajo }\end{array}$ & $\begin{array}{l}\text { Diplomado } \\
\text { Estudios de Normal Superior inconclusos } \\
\text { Diplomado }\end{array}$ \\
\hline Melisa & 47 & $\begin{array}{l}\text { Madre docente } \\
\text { Vocación }\end{array}$ & $\begin{array}{l}\text { Licenciada Educación Preescolar } \\
\text { Diplomado }\end{array}$ \\
\hline Cecilia & 23 & $\begin{array}{l}\text { Padre profesionista } \\
\text { Gusto por trabajar con niños } \\
\text { Gusto por el idioma }\end{array}$ & No indica (se toma como no) \\
\hline Keyla & 34 & $\begin{array}{l}\text { Padres docentes } \\
\text { Amistad con docente de inglés }\end{array}$ & $\begin{array}{l}\text { Licenciada en Relaciones Internacionales } \\
\text { Nivelación pedagógica } \\
\text { Maestría en docencia }\end{array}$ \\
\hline Matías & 24 & $\begin{array}{l}\text { Gusto por idiomas } \\
\text { Influenciado por } \\
\text { profesor de español } \\
\text { en secundaria }\end{array}$ & Licenciado en Docencia de Idiomas \\
\hline
\end{tabular}

Se observó que seis docentes participantes en este estudio incursionaron en la docencia por influencia de los padres o madres que, aunque no eran especialistas en idiomas, crearon una afición hacia la carrera en sus descendientes. Este grupo de docentes lleva a su práctica algunos saberes de sus familias así como expectativas en cuanto al rol sociocultural de la figura del maestro o maestra, el cual incide en su autoestima profesional.

A pesar de la influencia, solo cuatro docentes presentan formación en docencia de idiomas y cinco no cuentan con preparación en el área. No obstante, dentro de los argumentos mencionados se identificó que les fue necesario integrarse a una formación continua a través de programas de capacitación.

Asimismo, entre aquel grupo que presenta una preparación en el área, se identificaron tres tipos de formación que corresponden a licenciaturas afines. Estas son Licenciatura en Docencia de Idiomas, Licenciatura en Educación Preescolar y estudios de Normal Superior.

A partir de la identificación preliminar se buscó concretar conceptos creando la Tabla 2 para profundizar aún más en el núcleo de la formación inicial docente. Al descomponer las respuestas, partiendo desde lo más general a lo más específico, se pudo reducir la información, estructurarla y presentarla para luego extraer conclusiones particulares.

Se identifica una variedad de experiencias con el ingreso a la docencia. El ingreso se realiza a través de tres vías: la asignación de la plaza inicial, la selección libre de las direcciones o administraciones y de las propuestas del Sindicato Nacional de Trabajadores de la Educación (SNTE). La selección oficial de personal docente existe, al menos de manera somera; sin embargo, como evidencian los resultados, en el proceso de 
Ma. del Carmen Enriqueta Márouez-Palazuelos, et al. Los procesos de socialización de docentes de i...

contratación se involucran las relaciones personales más que la aplicación de estrategias institucionales para la selección del personal docente. Así, se manifiesta que la contratación de Olga y Valeria fue muy directa. En otros casos se tomó en cuenta su preparación profesional para la contratación. 
TABLA 2

Eje temático: Formación Docente - La experiencia en el campo y la inducción

\begin{tabular}{lll}
\hline Categoría & Entrevista & Muestra de evidencias en respuestas docentes \\
\hline Expectativa & ¿Cuál es tu proyecto & "Pues mi proyecto sería continuar asistiendo a todos los cursos, \\
profesional & a futuro como & talleres que me proporciona el sistema o a uno cuando pueda \\
& profesor de idiomas? & tomarlos, que se le dé importancia al maestro y tengamos las \\
& & herramientas para poder sacar adelante el segundo idioma, \\
& & porque no es fácil"
\end{tabular}

"Pues ahorita que se va a abrir una certificación CENNI quiero tomarla... pues lo que venga, los cursos que vengan"

"Mi proyecto personal es dar clases de regularización por las tardes a los niños que acuden a su horario normal que quieran reforzar, yo estaría trabajando con los más pequeños y hacerlo más personalizado"

"estudiar un doctorado. Ahorita estoy viendo la posibilidad de entrar a otra maestría, tengo la maestría en docencia, y estudiar un doctorado en mi área, negocios...si me visualizo en la docencia pero también he querido emprender algo en algún tipo de negocios..."

\begin{tabular}{ll}
\hline Ingreso al & ¿Cómo fue que \\
campo laboral & conseguiste tu \\
& empleo como docente \\
& en el Programa \\
& Nacional de Ingles?
\end{tabular}

"Pues como tengo la nivelación pedagógica, ya cubría interinatos en secundaria y un maestro al que cubrí que trabajaba también en primaria me dijo que requerían maestros para primaria"

"Lo que pasa es que en el sistema educativo había personas en los puestos, que estaban comisionadas que eran maestros con base... perdón, con plaza y dijeron que los iban a mandar a sus grupos y se iban a abrir espacios. Yo, este, metí mi curriculum y me dieron las clases" Supe a través de un conocido..."

"al inicio cuando yo regresé de Estados Unidos no estaba estudiando no tenía trabajo entonces empecé en un jardín de niños y un amigo me consiguió trabajo para que hiciera algo por mientras que entraba a estudiar, me fui a un jardín particular. me dieron trabajo y estuve trabajando con niños de preescolar y fue por eso que me interese en lo que es la educación preescolar, me gustó la forma en que la maestra trabajaba ahí y como yo daba inglés y miraba como los niños aprendían con canciones y todo y por eso me dio por ese lado a trabajar en el sistema"

¿Porque te dieron el "El director estaba desesperado porque no sabía qué hacer con trabajo? ¿Fue fácil o esos grupos de inglés, porque no pagan mucho, no tienen muchos difícil? derechos y pues yo me animé

"Fui a ISEP, me entrevistó la coordinadora del inglés en primarias, fue relativamente fácil, porque pude conseguir el trabajo"

\begin{tabular}{lll}
\hline Capacitación & $\begin{array}{l}\text { ¿Recibes capacitación } \\
\text { del programa? }\end{array}$ & $\begin{array}{l}\text { "La capacitación la hemos pagado nosotros, así que sería muy } \\
\text { bueno que nos apoyaran con el } 50 \% \text { de la actualización. }\end{array}$
\end{tabular}

Si, a mí a cada rato me invitan a cursos, de hecho el diplomado que tomé lo pagó... eh.. no sé si la coordinación de inglés pero ella me mandó allá. Un diplomado que si no lo tomábamos nos iban a cobrar los \$10,000.00 pero yo ni me fijé en eso, dije diplomado de la universidad de San Diego, lo tomo..."

Retos ¿Cuáles son los retos "Ahorita como está lo de los maestros que está siendo denigrada que enfrentas? la profesión. Esta profesión es muy bonita pero está siendo atacada por los medios de comunicación. Esta situación se ha venido a partir de la reforma educativa. Yo creo que el problema no es educativo es laboral...deberían revisar los planes y programas, si están fallando entonces ver que le puedo dar al maestro, que necesita para desarrollar su trabajo. Eso es lo que se tuviera que cambiar. Además la situación se complica pues se están incluyendo a los niños con capacidades especiales. 
Nota: Categorización de las entrevistas semiestructuradas respecto a la formación docente.

En esencia, los sujetos participantes mencionaron experiencias propias correspondientes a su formación inicial y su formación continua, en donde el personal docente comprometido con su desarrollo profesional continuo integra una variedad de experiencias que permiten un desarrollo efectivo. De allí, docentes como Matías encuentran satisfacción en su desempeño y María, se empeña en adscribirse a una formación continua.

Tomando el segundo rubro de este estudio, la construcción de la identidad profesional, se evidenciaron los inicios a partir del ingreso al espacio escolar, ya que tras la inducción (o la falta de la misma) llega la búsqueda de mejores condiciones de contratación y de trabajo. Keyla en su comentario sitúa los primeros rasgos profesionales:

"Fíjese que yo pienso que no se le da el valor que nos merecemos por muchas partes..." (KEYLA)

Así también, en el caso de docentes que laboran en el Programa Nacional de Inglés, se percibe la falta de una inducción apropiada a su ingreso al contexto laboral.

Para los maestros y maestras en servicio, la falta de reconocimiento, donde la importancia de tener o no estudios parece diluirse, suele sentirse como extranjeros en un mundo extraño (Johnston y Ryan, 1983) y sufren un choque con la realidad afrontando el inicio de su práctica docente con apoyo del personal directivo o sin este.

Se teoriza que la forma en que cada docente es percibido por sus colegas, personal directivo y de coordinación al ingreso a su contexto laboral es de suma importancia para la construcción y reconstrucción continua de su identidad. Según Baró (1976), estos primeros momentos de su ingreso al contexto de trabajo pueden tornarse como una primera experiencia significativa que puede valorarse positiva o negativamente e impactar su trayectoria y su labor docente. Definitivamente, tal situación incidirá en la forma en que se empiece a relacionar con el contexto, y podría desarrollar, quizás, un sentimiento de inseguridad y de aislamiento.

En el caso de docentes que pertenecen al Programa Nacional de Inglés, esa primera experiencia que les impacta es recurrente. Desafortunadamente, el contexto de escuelas de tiempo completo en las que laboran promueve este hecho, ya que las clases de inglés dan inicio por la tarde cuando las clases regulares terminan. Por ello, este personal docentes tiene pocas oportunidades de interaccionar con sus colegas de otras disciplinas.

Identidad profesional. Tras explorar la inducción a la docencia vinculada con los saberes específicos de su profesión, se procedió con la entrevista programada con la aplicación de una secuencia de preguntas más puntuales cuyos resultados recopilados en la Tabla 3 ayudan a dimensionar y estructurar este fenómeno de la identidad. A través de un proceso exploratorio se compartió una serie de dificultades variadas que colindan con su imagen profesional y hasta su autoestima. Dichas dificultades se encuentran vinculadas a su situación laboral, su realidad cultural, dominio de competencias e interacciones con sus pares. 
TABLA 3

Eje temático: Identidad profesional - Saberes específicos de su profesión

\begin{tabular}{|c|c|c|}
\hline Categoria & Entrevista & Muestra de evidencias en respuestas docentes \\
\hline $\begin{array}{l}\text { Experiencia } \\
\text { docente }\end{array}$ & $\begin{array}{l}\text { ¿Cuánto } \\
\text { tienes dando } \\
\text { clases de } \\
\text { inglés? }\end{array}$ & $\begin{array}{l}\text { Haydee. } 10 \text { años } \\
\text { Olga.- } 8 \text { años } \\
\text { Melisa. } 6 \text { años } \\
\text { Maria. } 2 \text { años } \\
\text { Valeria }- \text { no indica }\end{array}$ \\
\hline $\begin{array}{l}\text { Creencias, } \\
\text { percepción }\end{array}$ & $\begin{array}{l}\text { ¿Cuáles } \\
\text { experiencias } \\
\text { positivas } \\
\text { has tenido } \\
\text { en el } \\
\text { programa? }\end{array}$ & $\begin{array}{l}\text { "...siempre trato de hacer lo mejor ...tanto para los alumnos como para mi, de } \\
\text { que no.... ¿Cómo decirlo?...que a la vez que están aprendiendo...se diviertan y } \\
\text { agarren ese gusto por Idiomas...no solo uno sino varios idiomas." (MELISA) } \\
\text { "...ahi en la escuela donde trabajo... hay maestros que se esfuerzan } \\
\text { muchisimo...e igual es solamente una [mala] imagen que se ha tenido por } \\
\text { ciertas personas y más en inglés..." (VALERLA) } \\
\text { "... la verdad, ser maestro pues, ¿No es cualquier cosa, no?, pues como } \\
\text { muchos dicen...es hacerlo de doctor, de psicólogo, de... hasta incluso de } \\
\text { padres...tratando de sacar adelante al niño." (HAYDEE) } \\
\text { "...fue muy interesante cuando entramos a los diplomados, a los talleres, } \\
\text { porque pues ahi nos dan herramientas." (CECILIA) }\end{array}$ \\
\hline & $\begin{array}{l}\text { ¿Cuál es tu } \\
\text { opinión del } \\
\text { programa } \\
\text { de inglés en } \\
\text { primaria? }\end{array}$ & $\begin{array}{l}\text { "...no sé ahora que voy a la primaria y veo toda la dinámica, si ha cambiado } \\
\text { más mi idea ('sprofesión con futuro?)... Si, si tiene un futuro..." (MATIAS) } \\
\text { "La sociedad no visualiza la importancia que tiene el inglés, el } 50 \% \text { no } \\
\text { reconocen esa importancia." (MELISA) } \\
\text { "...también vemas, si, si nos dan trabajo, pero no hay mucho apoyo." (SILVIA) } \\
\text { "Pues mi opinión, es que ha ido de más a menos en todos los aspectos (ipor } \\
\text { qué?) Pues, porque antes tentamos capacitaciones..." (OLGA) } \\
\text { "...trabajamos con más niños, ehh con menos tiempo y con igual paga, } \\
\text { entonces, no tenemos ya las capacitaciones..." (VALERIA) }\end{array}$ \\
\hline Académicos & $\begin{array}{l}\text { ¿Cuáles han } \\
\text { sido tus } \\
\text { logros en la } \\
\text { docencia de } \\
\text { idiomas? }\end{array}$ & $\begin{array}{l}\text { "...estar a la vanguardia preparándonos, investigando... } \\
\text { retroalimentándonos; es muy importante siempre estar aprendiendo porque } \\
\text { uno no termina realmente." (MARLA) } \\
\text { "...pues yo tuve la oportunidad de hacer un intercambio y lo hice aqui mismo } \\
\text { en México, lo hice en Guadalajara..." (KEYLA) } \\
\text { "...me invitan mucho la Directora y la ATP junto con la Coordinadora de } \\
\text { inglés, me invitan mucho a tomar cursos, jcómo se dice? A actualizarme todo } \\
\text { eso, pues, ahora asi que sin sonar, ...muy alardeante...pues ven mi trabajo o } \\
\text { ven mis ganas la verdad, ...y quieren que, que lo haga..." (CECILIA) } \\
\text { "...bien, tengo muy buena relación dinámica con, con mis grupos siendo que } \\
\text { antes les tenía miedo y bueno son, son sólo niños...pero no la verdad ahorita } \\
\text { si me está gustando mucho, siento que eso fue lo que logre." (SILVIA) }\end{array}$ \\
\hline
\end{tabular}

Nota: Categorización de las entrevistas semiestructuradas respecto a la identidad profesional en la construcción y el reconocimiento de una identidad propia. 
Al haber incursionado en la institución educativa, el personal docente ubica sus principales puntos de referencia para adecuarse a su contexto como: sus estudiantes, sus colegas, las autoridades, los padres y madres de familia, así como el programa. Se proyectan en cada uno de estos grupos en el proceso de autoconstruir su identidad y su rol en la institución.

En la Tabla 3 se evidencia esta autoconstrucción tanto a través de sus creencias personales como de su rol docente. Luego, al cuestionarles primeramente sus creencias (personales) el personal docente de inglés presenta capacidades, cualidades y logros que le son propias. Se muestra como un participante en el quehacer escolar con ideales, proyectos, motivaciones y circunstancias particulares que le definen. En su rol docente, se evidencia como agente que ejecuta numerosas acciones, orienta, dirige y guía la interacción de su alumnado.

La Tabla 4 identifica estos rasgos de proyección en las personas docentes participantes como: el vínculo que se establece entre lo que sabe y la práctica docente, el conocimiento que tiene sobre sí y sobre el mundo que le rodea, su preparación lingüística, su puesta en práctica de la enseñanza, su articulación entre formación e investigación y, finalmente, su capacidad de adecuarse al contexto. 
TABLA 4

Eje temático: Identidad profesional - Proyección de la identidad docente

\begin{tabular}{|c|c|c|}
\hline Cate & Entrevista & Muestra de evidencias en respuestas docentes \\
\hline & $\begin{array}{l}\text { ¿Te sientes } \\
\text { profesional en } \\
\text { tu área? }\end{array}$ & $\begin{array}{l}\text { "Si, si me he sentido profesional porque me he capacitado, he tomado } \\
\text { cursos, estudio de forma autónoma y...sigo estudiando" (KEYLA) } \\
\text { " "No, porque siento que me hace mucha falta experiencia. Necesito } \\
\text { actualizarme practicar más con la enseñanza." (MARIA) } \\
\text { "Si, principalmente si yo me pongo como una tercera persona siento que } \\
\text { puedo admirar mis planeaciones, y las actividades que tengo, siento } \\
\text { que tengo una buena facilidad para...como se dice, para saber cuál es el } \\
\text { objetivo y ver que herramientas innovadoras adaptarle..." (SILVIA) }\end{array}$ \\
\hline \multirow[t]{2}{*}{ Profesional } & $\begin{array}{l}\text { ¿Cuáles son } \\
\text { los retos que } \\
\text { enfrentas como } \\
\text { docente? }\end{array}$ & $\begin{array}{l}\text { "...si pienso que necesito actualizarme y tal vez este... pues no se... practicar } \\
\text { más con la enseñanza." (MARIA) } \\
\text { "..pues creo que aún algunas personas aún consideran que un docente... } \\
\text { puede ser cualquier persona." (SILVIA) } \\
\text { "Si... siempre... ha... nos incluyen... somos dos maestros de inglés...y } \\
\text { a pesar de que apenas se acaba de incluir el inglés...siempre nos están } \\
\text { pidiendo opinión...eh. cosa que se va a hacer en la escuela piden ayuda" } \\
\text { MATIAS) } \\
\text { "Fue un reto para mi muy grande el ponerme delante de ellos y darles } \\
\text { clases: entonces mi logro fue que después de esa experiencia puedo pararme } \\
\text { ahorita...y me está yendo bastante bien...." (MELISA) }\end{array}$ \\
\hline & $\begin{array}{l}\text { ¿Cómo estos } \\
\text { procesos } \\
\text { determinan tu } \\
\text { identidad como } \\
\text { profesional? }\end{array}$ & $\begin{array}{l}\text { "Que mi desempeño ha impactado en el entorno escolar." (SILVIA) } \\
\text { "Pues... yo creo que, si he tenido un tipo de efecto porque, si me contaban } \\
\text { que los maestros a veces les ponian películas, les daban las respuestas, } \\
\text { entonces, cuando ven otro tipo de trabajo... van tomando cada vez más, } \\
\text { más gusto a las cosas." (KEYLA) } \\
\text { "...como yo tengo primero y segundo grado, pues, adecuar lo básico, lo que } \\
\text { daba yo antes, pues jalé muchas cosas que tenia, y a buscar en internet, a } \\
\text { buscar cosas para poder dar lo que uno tiene que dar..." (HAYDEE) } \\
\text { "...podemos manejarlo. O sea uno, porque ya por lógica...uno ya tiene esa } \\
\text { pedagogía porque la he estudiado..." (MARIA) }\end{array}$ \\
\hline $\begin{array}{l}\text { Impacto } \\
\text { social }\end{array}$ & $\begin{array}{l}\text { ¿Has tenido } \\
\text { problemas? }\end{array}$ & $\begin{array}{l}\text { "Pues hasta ahorita yo me siento como desfasada, con ellos porque no, } \\
\text { no le veo la relevancia discutir...este, o hablar mal de una persona...yo me } \\
\text { siento este, ajá, como que no encajo mucho..." (SILVIA) } \\
\text { "El maestro de inglés, no tiene como obligación asistir a los consejos } \\
\text { técnicos pero, aun así los directores requieren que vayan...entonces muchas } \\
\text { veces el maestro de inglés está ahí pero no lo toman en cuenta...(MARIA) }\end{array}$ \\
\hline Personales & $\begin{array}{l}\text { ¿Cuáles aon } \\
\text { los retos que } \\
\text { enfrentas? }\end{array}$ & $\begin{array}{l}\text { "...(del docente en México) un tanto dificil en todos los aspectos, en cuanto } \\
\text { al aspecto laboral en cuanto al aspecto económico como que estamos un } \\
\text { poquito marginados..." (MARIA) }\end{array}$ \\
\hline En el aula & & "Yo los hago reflexionar sobre la importancia del inglés..." (MATIAS) \\
\hline $\begin{array}{l}\text { Del } \\
\text { programa }\end{array}$ & $\begin{array}{l}\text { ¿Si pudieras } \\
\text { hacer cambios } \\
\text { al programa, } \\
\text { cuáles serían? } \\
\text { ¿Porque? }\end{array}$ & $\begin{array}{l}\text { "..corroboraría que el maestro estuviera capacitado para la enseñanza del } \\
\text { inglés... faltaría checar esa parte de los linesmientos y la capacitación de } \\
\text { los maestros." (MARIA) } \\
\text { "Creo que eso si le faltaría...tener maestros preparados y segundo que } \\
\text { hubiera un contacto con los directores, las coordinaciones para ver qué } \\
\text { onda con el programa, ver si les ha funcionado y si no porqué..y establecer } \\
\text { productos o metas acordes...no nada más que suenen en papel." (CECILIA) }\end{array}$ \\
\hline
\end{tabular}


Nota: Categorización de las entrevistas semiestructuradas respecto a la identidad profesional en la construcción de un proyecto profesional.

Categorización de las entrevistas semiestructuradas respecto a la

identidad profesional en la construcción de un proyecto profesional.

La percepción del grupo de docentes participantes que han incursionado en el escenario del Programa Nacional de Inglés sobre su proyecto a futuro como docentes, su entrada al campo laboral, y su capacitación continua, encuentra relación directa con su percepción de la complejidad de la práctica educativa docente, su desarrollo en el proceso de enseñanza-aprendizaje y su formación docente como actividad permanente en perfeccionamiento.

Menciona, una parte del grupo de docentes en la entrevista que en ocasiones se ha sentido aislado por el clima general, clima operativo y clima interno de la institución en donde labora.

La visión que perciben en relación con el contexto académico, es expresada por (MARIA) en los siguientes términos:

"El maestro de inglés, no tiene como obligación asistir a los consejos técnicos pero, aun así los directores requieren que vayan... entonces muchas veces el maestro de inglés está ahí pero no lo toman en cuenta..."

Sin embargo, no todos los escenarios muestran la misma situación como se refleja en los siguientes comentarios:

"Si... siempre... ha... nos incluyen... somos dos maestros de inglés...y a pesar de que apenas se acaba de incluir el inglés... siempre nos están pidiendo opinión...” (MATIAS)

En cuanto a la pregunta, ¿cómo estos procesos determinan tu identidad como profesional?, se puede confirmar que la construcción de la identidad se conjuga en lo público y lo privado, lo personal y lo profesional (ver Tabla 4). Los grupos docentes, al insertarse en un contexto laboral, cuentan con una identidad previamente construida desde sus experiencias personales, influenciados quizás por personas significativas en su vida familiar o en su trayectoria como estudiantes de niveles previos y, finalmente, en su paso por la formación docente (Tablas 1 y 3). Sin embargo, como afirman Norton (1995) y Van Dijk (1999), esta identidad no es permanente, es un proceso dinámico que será influenciado por las experiencias positivas o negativas a las que se enfrente en su entorno laboral.

Muchas instituciones formadoras de docentes presuponen que su estudiantado egresado cuenta con las habilidades suficientes requeridas en la profesión de la docencia. La realidad muestra dos posicionamientos. Por un lado, algunos nuevos grupos de docentes consideran ser profesionales en el área y otros aún se encuentran en la construcción de esa identidad. La práctica cotidiana les proporciona el ámbito para reflexionar sobre sus habilidades y áreas de oportunidad y, de esa manera, van construyendo y reconstruyendo en forma continua su identidad profesional (ver Tabla 3).

En relación con su sentir como profesionales de la docencia, se puede observar que varias personas del grupo depositan su valía en la preparación continua dentro de su profesión y otras la encuentran en su dedicación hacia sus estudiantes y en la respuesta de su alumnado a su compromiso. Por otra parte, los retos y las expectativas profesionales que enfrentan en su cotidianeidad, promueven también su tránsito hacia ese sujeto docente ideal que tienen en su imaginario y el cual anhelan alcanzar.

Cultura organizacional. Al integrar un tercer rubro al presente análisis, el de la cultura organizacional, se conjugan el amalgamiento entre los elementos que preparan al personal docente de manera inicial y aquellos procesos que le dan identidad con las características de la institución educativa donde se integra laboralmente. En este rubro se evidencia, entonces, la institución educativa donde cada docente acepta regirse por los elementos que le dan identidad: su ideología, sus creencias, valores y objetivos; así como las acciones que como resultado emerjan de la integración de todos sus elementos, los cuales posicionan y caracterizan al personal docente que labora en ella. 
Este proceso de amalgamiento entre los elementos que le dan identidad profesional al personal docente y los elementos que caracterizan la institución como un ente puede ser parte de un proceso de socialización docente directa o puede resultar en un constante rechazo de ambos al proceso de socialización e integración (Ramírez et al., 2012). La bibliografía revisada, y en particular Benegas (2002), conceptualizan cómo se entremezclan las expectativas personales, las experiencias institucionales e históricas-culturales en un permanente proceso de socialización profesional. La información obtenida a partir de las entrevistas semiestructuradas realizadas muestran este proceso.

“...yo me siento este, ajá, como que no encajo mucho...” (SILVIA).

“...entonces muchas veces el maestro de inglés está ahí pero no lo toman en cuenta...los maestros de base se ponen a discutir los problemas que tienen en sus grupos, pero al maestro de inglés muchas veces no lo consideran, está ahí escuchando pero no lo toman en cuenta..." (MARÍA).

De la misma manera, la incertidumbre y ambigüedad existente en las normas que rigen la prestación del servicio que desarrolla el personal docente de idiomas, hasta ahora. La formación académica, el manejo del idioma, la profesión, la contratación, la obligatoriedad de la asignatura, el número de horas y horario, entre otros elementos, impiden que el maestro o maestra de lenguas pueda establecer relaciones estrechas con los elementos y conductas que definen las escuelas en donde laboran (Ramírez et. al., 2012).

En consecuencia, los grupos de docentes de este estudio describen retos que se ubican en las vertientes: a) atención al niño y a la niña; b) desarrollo de una educación integral; c) carencia y falta de infraestructura adecuada para su área y reconocimiento a su profesión (ver Tabla 5). 
TABLA 5

Eje temático: Cultura organizacional

\begin{tabular}{lll}
\hline Categoria & Entrevista & Muestra de evidencias en respuestas docentes \\
\hline Expectativa & ¿Cuál es tu proyecto & "Pues mi proyecto sería continuar asistiendo a todos los cursos, \\
profesional & a futuro como & talleres que me proporciona el sistema o a uno cuando pueda \\
& profesor de idiomas? & $\begin{array}{l}\text { tomarlos, que se le dé importancia al maestro y tengamos las } \\
\end{array}$ \\
& & herramientas para poder sacar adelante el segundo idioma, \\
& porque no es fácil"
\end{tabular}

"Pues ahorita que se va a abrir una certificación CENNI quiero tomarla... pues lo que venga, los cursos que vengan"

"Mi proyecto personal es dar clases de regularización por las tardes a los niños que acuden a su horario normal que quieran reforzar, yo estaría trabajando con los más pequeños y hacerlo más personalizado"

"estudiar un doctorado. Ahorita estoy viendo la posibilidad de entrar a otra maestría, tengo la maestría en docencia, y estudiar un doctorado en mi área, negocios...si me visualizo en la docencia pero también he querido emprender algo en algún tipo de negocios..."

\begin{tabular}{ll}
\hline Ingreso al & ¿Cómo fue que \\
campo laboral & conseguiste tu \\
& empleo como docente \\
& en el Programa \\
& Nacional de Ingles?
\end{tabular}

"Pues como tengo la nivelación pedagógica, ya cubría interinatos en secundaria y un maestro al que cubrí que trabajaba también en primaria me dijo que requerían maestros para primaria"

"Lo que pasa es que en el sistema educativo había personas en los puestos, que estaban comisionadas que eran maestros con base... perdón, con plaza y dijeron que los iban a mandar a sus grupos y se iban a abrir espacios. Yo, este, metí mi curriculum y me dieron las clases" Supe a través de un conocido..."

"al inicio cuando yo regresé de Estados Unidos no estaba estudiando no tenía trabajo entonces empecé en un jardín de niños y un amigo me consiguió trabajo para que hiciera algo por mientras que entraba a estudiar, me fui a un jardín particular, me dieron trabajo y estuve trabajando con niños de preescolar y fue por eso que me interese en lo que es la educación preescolar, me gustó la forma en que la maestra trabajaba ahí y como yo daba inglés y miraba como los niños aprendían con canciones y todo y por eso me dio por ese lado a trabajar en el sistema"

¿Porque te dieron el "El director estaba desesperado porque no sabía qué hacer con trabajo? ¿Fue fácil o esos grupos de inglés, porque no pagan mucho, no tienen muchos difícil? derechos y pues yo me animé

"Fui a ISEP, me entrevistó la coordinadora del inglés en primarias, fue relativamente fácil, porque pude conseguir el trabajo"

Capacitación ¿Recibes capacitación "La capacitación la hemos pagado nosotros, así que sería muy del programa? bueno que nos apoyaran con el $50 \%$ de la actualización.

Si, a mí a cada rato me invitan a cursos, de hecho el diplomado que tomé lo pagó... eh.. no sé si la coordinación de inglés pero ella me mandó allá. Un diplomado que si no lo tomábamos nos iban a cobrar los \$10,000.00 pero yo ni me fijé en eso, dije diplomado de la universidad de San Diego, lo tomo..."

Retos ¿Cuáles son los retos "Ahorita como está lo de los maestros que está siendo denigrada que enfrentas? la profesión. Esta profesión es muy bonita pero está siendo atacada por los medios de comunicación. Esta situación se ha venido a partir de la reforma educativa. Yo creo que el problema no es educativo es laboral...deberían revisar los planes y programas, si están fallando entonces ver que le puedo dar al maestro, que necesita para desarrollar su trabajo. Eso es lo que se tuviera que cambiar. Además la situación se complica pues se están incluyendo a los niños con capacidades especiales. 


\begin{abstract}
Nota: Conceptualización y categorización de las entrevistas semiestructuradas respecto a la cultura organizacional constituida por el clima general (aspectos sociales, legales, económicos y tecnológicos), clima operativo (administrativo, laboral) y clima interno (fuerzas internas de la institución).
\end{abstract}

Las políticas educativas, inclusión escolar y programas en educación básica se han modificado, por lo que la capacitación docente y dotación de materiales y equipos a las escuelas se ha llevado a cabo, en los últimos años. Sin embargo, el programa de inglés, aunque ha sufrido adecuaciones, no ha generado que los elementos que giran a su alrededor sufran transformaciones o sean objeto de capacitación: docentes y mejoramiento; materiales y equipo. Ello, como se menciona anteriormente, crea un estado de incertidumbre, inseguridad y desapego que impacta los procesos de socialización del personal docente de idiomas en la escuela y de su identificación con el centro escolar; se construye, de esta manera, una barrera que impide a quien enseña lenguas integrarse a la culturas organizacional de la institución educativa.

Esto lo podemos identificar en los comentarios expuestos por los maestros y maestras en las entrevistas:

De estudiantes

"Uno de los retos que enfrento es la indisciplina...otro es el nivel de inglés que realmente los niños no tenían nada, ahora ya tienen un poco..." (MATIAS)

“Tener niños con capacidades diferentes. Avanzar con estos niños ha sido muy difícil porque en un grupo de 30 he tenido 5 con esa situación...Creo que necesitamos capacitación de USAER sólo para maestros.” (KEYLA)

Del desarrollo integral

“...tratar de vincular, como se dice, los aprendizajes sociales a la clase de inglés." (SILVIA)

De la infraestructura

"Hay muchos materiales que pueden estar en contacto con la tecnología y no la tenemos...porque no tenemos, una, laboratorio donde podamos experimentar todo eso pues al estar interactuando con la tecnología junto con el idioma, es más fácil aprender inglés." (MELISA)

Del reconocimiento profesional

“me gustaría también se le reconociera más al maestro de inglés...como parte del equipo de trabajo...en cuanto a lo laboral... que fueran reconocidos verdaderamente porque igual hacen la misma labor con los niños, son parte de la formación de los niños, entonces creo yo que ...si sería bueno que los apoyaran un poquito." (HAYDEE).

\title{
ConCLUSIón
}

El estudio realizado nos permitió dar respuesta a la pregunta: ¿Quiénes son los sujetos docentes que colaboran en el programa nacional de inglés? A través de las respuestas que nos compartieron se permitió conocer procesos de integración de docentes de la materia de inglés en Ensenada, Baja California, México, así como la cultura y las dinámicas de trabajo de la institución en la que laboran.

También, el estudio muestra que la metodología de investigación utilizada permitió dar voz al personal docente y extraer información referente a las experiencias personales vividas por el grupo entrevistado. Específicamente, se logró exponer la opinión, el pensamiento, las creencias, sentimientos e interpretación de docentes de inglés sobre el proceso de socialización al que se ven expuestos al ingresar a las escuelas de tiempo completo que tienen programa de inglés, y se generó un conocimiento profundo sobre el tema.

Así se encontró que, a pesar de que el personal docente presenta un grado académico de licenciatura como obligatorio para el perfil docente que imparte la materia de inglés en el nivel educativo básico, aún se privilegia la condición de manejo del idioma y el acceso al sistema en docentes con una formación académica carente del nivel deseado.

En relación con la pregunta: ¿cómo estos procesos determinan su identidad como profesional? Las respuestas de docentes muestran una falta de reconocimiento como profesionales en un área que otorga poco valor a la materia de inglés en el plan de estudios de educación básica y continua en Baja California, México. 
El personal docente de inglés es recibido con cierta indiferencia por los cuadros directivos y colegas al ingresar al centro educativo donde labora. Esta situación afecta el proceso de socialización del profesorado de inglés dentro de la escuela, pues favorece el aislamiento y el trabajo individual, genera que la autoestima y el autoconcepto profesional en la enseñanza de inglés se vea disminuido y provoca que surjan sentimientos de inseguridad en su papel docente.

De la misma manera, su identidad profesional de docente de idiomas es difícil de definir y afianzar, dadas las condiciones laborales y sociales a las que se enfrenta. Esto concuerda con lo expresado por el francés Dubar (1991), quien pone en el centro de la construcción de la identidad los vínculos (existentes o inexistentes) entre sujetos e instituciones sociales.

Docentes participantes en el estudio identificaron claramente las necesidades en torno a su identidad profesional. Explicaron que las necesidades son en torno a la capacitación para la enseñanza del inglés y al acercamiento con otros sujetos participantes en el ámbito escolar.

Así también, la incertidumbre y la ambigüedad existente en torno al servicio que presta el personal docente de inglés impide un buen clima organizacional. Es difícil establecer relaciones positivas con otros elementos de la organización escolar, tanto de tipo personal, como en torno al programa y a los recursos, ya que no existen normas bien definidas

\section{Recomendaciones}

Para asegurar la calidad de la educación en todos los ámbitos del sistema educativo, pero mayormente en el área que nos ocupa -la enseñanza del inglés en las escuelas primarias públicas-, es necesario que se considere atender el desarrollo organizacional, tanto desde las variantes de consultoría externa (coordinación) como interna (dirección). También, se requiere fijar la atención en desarrollar formas eficientes y holísticas que garanticen el desarrollo del programa a partir de la potenciación de fenómenos y procesos estratégicos que atiendan su buen funcionamiento.

De igual manera, el personal docente de inglés, como integrante del fenómeno educativo, se desarrolla en una sociedad donde está ampliamente representada la organización escolar. Su aportación a las relaciones humanas, las actividades laborales, las actividades sociales y la vida cotidiana, en general, depende de la calidad de su formación, reconocimiento y buena organización.

Para lograr este fin es necesario que se crean espacios para que los cuerpos docentes que laboran en el Programa Nacional de Ingles se identifiquen e intercambien experiencias; para mejorar, por un lado, su desarrollo profesional y, por otro, lograr reconocerse como pares que realizan una misma labor docente con otras personas integrantes del sistema educativo. Todo esto promovería su desarrollo profesional, su identidad y pertinencia, y su acercamiento a la cultura organizacional.

Afirma Borko (2004) que uno de los factores que puede promover el desarrollo profesional es el establecimiento de comunidades de práctica. Estas comunidades aportarían al fortalecimiento de la identidad de docentes de inglés que laboran en el Programa Nacional de Inglés y favorecería la construcción de espacios de crecimiento y relaciones de confianza que brindan apoyo a la construcción de su reconocimiento profesional.

\section{ReFERENCIAS}

Baro, M. (1976). Docencia, investigación y proyección social. Principios y orientaciones. APA Psyc Net. Recuperado de: https://doi.org/10.1037/a002 6866

Bazdresch, M. (2006). La intervención en la práctica educativa, en R. C. Perales-Ponce (Coord.), La significación de la práctica educativa (pp. 55-70). México: Innovación Educativa. 
Balderas, I. (2013). Propuesta de guion de entrevista para el estudio de la identidad docente. Revista Latinoamericana de Metodología de la Investigación Social, 6. Recuperado de: http://www.relmis.com.ar/ojs/index.php/relmis/ article/view /84

Benegas, M. A. (2002). El proceso de socialización profesional: Una mirada desde la cultura institucional. Revista Argentina de Enseñanza de la Ingeniería, 3(5), 61-66. Recuperado de http://www.ing.unrc.edu.ar/raei/ archivos/img/arc_2011-11-23_21_01_32-56.pdf

Bolívar, A. y Domingo, J. (2006). La investigación biográfica y narrativa en Iberoamérica: Campos de desarrollo y estado actual. Forum for Qualitative Social Research, 7(4). doi: http://dx.doi.org/10.17169/fqs-7.4.161

Borko, H. (2004). Professional Development and Teacher Learning: Mapping the Terrain. Educational Researcher, 33(8). doi: https://doi.org/10.3102/0013189 X033008003

Brito, A., Araneda N., Hernández J. y Lorca, J. (2005). Inducción profesional docente. Estudios Pedagógicos, 31(1), 51-62. Recuperado de https://scielo.conicyt.cl/scielo.php?script=sci_arttext\&pid=S0718-070520050 00100003

Calderón, D. (2015). La política educativa actual del inglés en México. Sorry, el aprendizaje del inglés en México. Mexico City: Mexicanos Primero.

Corley, E. L. (1998). First-year teachers: Strangers in strange lands [Docentes novatos: Extraños en tierras extrañas]. Ponencia presentada en la Junta Anual de la Midwestern Educational Research Association, Chicago, IL. (ERIC Servicio de Reproducción de Documentos N.o ED 424216).

Danielewicz, J. (2001). Teaching selves: Identity, pedagogy, and teacher education [Educándose a sí mismos: Identidad, pedagogía y formación docente]. Albany, NY: State University of New York Press.

Davini C. (1995). Formación docente en cuestión: Política y pedagogía. Buenos Aires. Paidós.

Day, C. (2005). Formar docentes. Madrid: Narcea.

Díaz Barriga, Á. e Inclán, C. (2001). El docente frente a las reformas educativas. Sujeto o ejecutor de proyectos ajenos. Revista Iberoamericana de Educación, 25. Recuperado de https://rieoei.org/historico/documentos / rie25a01.htm

Dubar, C. (1991). La socialisation. Construction des identités sociales et professionnelles [La socialización. Construcción de identidades sociales y profesionales]. Paris: Armand Colin.

Fierro L. y Martínez L. (2015). Configuraciones sobre la construcción de la identidad profesional de los profesores de inglés como lengua extranjera de escuelas primarias públicas. Memoria del Congreso Internacional de Idiomas 2013. México: UABC.

Gairín, J. y Armengol, C. (2003). Estrategias de formación para el cambio organizacional. Barcelona: Cisspraxis.

Gobierno del Estado de Baja California (2016). Implementa Gobierno del Estado programa de ingles conversacional en escuelas primarias de Baja California. Portal Noticias: Gobierno del Estado.

González, M. (1995). Formación docente: Perspectivas desde el desarrollo del conocimiento y la socialización profesional. Barcelona: PPU.

H. Cámara de Diputados, LX Legislatura (2007). Plan Nacional de Desarrollo 2007-2012 escenarios, programas e indicadores. Programa Sectorial de la Secretaria de Educación Publica 2007-2012. México D.F.

Imbernón, F. (2007). La formación y el desarrollo profesional del profesorado. Hacia una nueva cultura profesional (7a ed.). España: Grao.

Johnston, J. y Ryan, K. (1983). Research on the Beginning Teacher: Implications for Teacher Education. En K. R. Howey y W. Gardner (Eds.), The Education Teachers. A look ahead (pp. 136-162). New York: Longman.

Kinnear, T. y Taylor, J. (1998). Investigación de mercados: Un enfoque aplicado (5ª ed.). México: McGraw-Hill.

LaPointe, K. (2011). Moral struggles, subtle shifts: Narrative practices of identity work in career transitions (Tesis de doctorado). Universidad de Aalto, Aalto, Finlandia.

Latorre, M. y Blanco, E. (2007). Algunos conceptos clave en torno a las creencias de los docentes en formación. Docencia e investigación, 32(17), 1-19. Recuperado de: https://ruidera.uclm.es/xmlui/bitstream/ handle/10578/8093/Algunos_conceptos_clave_en_torno_a_las_creencias_d.pdf 
Márquez. C. (2011). Perspectiva de la enseñanza del inglés en las primarias públicas de Baja California. Universidad de Sonora. Memoria electrónica Foro Nacional sobre Enseñanza de Lenguas Extranjeras. Hermosillo, Sonora.

Miles, M. y Huberman, A. M. (1984). Qualitative data analysis, a source book of new methods. Beverly Hills: Sage Publications.

Norton, B. (1995). Social identity, investment, and language learning. TESOL Quarterly, 29(1), 9-31. doi: https:// doi.org/10.2307/3587803

Pérez, Y. (2007). Problemas epistemológicos en el proceso de formación de profesores de lenguas extranjeras. Revista Iberoamericana de Educación, 4(12), 1-5.

Peteiro, L. M. (2007). La relación entre cultura organizacional e instituciones educativas. Recuperado de https:// www.gestiopolis.com/la-relacion-entre-cultura-organizacional -e-instituciones-educativas

Plan de Desarrollo Nacional 2007-2012 - Programa Nacional de Inglés en Educación Básica. Segunda Lengua: Ingles. (2011 primera ed. electrónica). Recuperado de http://www.pnieb.net/inicio.html

Ramírez, J. L., Pamplón, E. N. y Cota, S. (2012). Problemática de la enseñanza del inglés en las primarias públicas de México: Una primera lectura cualitativa. Revista Iberoamericana de Educación, 60(2), p. 4-10.

Saiz, Á. (2014). La investigación biográfico-narrativa como estrategia de formación docente. En J. J. Maquillón y J. I. Alonso (Eds.), Experiencias de innovación y formación en educación (p. 23-33). Murcia: Edit.um.

Subsecretaria de Educación Básica. (2016). Dirección General de Desarrollo Curricular. Programa Nacional de Inglés. Recuperado de http://www.dgdc.sep.gob.mx/dgdc2016/progfederales/dgdc_prog_fed_PRONI.htm

Subsecretaria de Educacion Basica. Sistema Educativo Estatal (2017). Actualización, Programa de Educación de Baja California 2015-2019. COPLADE.

Urrutia, F. (2013). ¿Descuidar el corazón? Comentarios al Plan Nacional de Desarrollo 2013-2018, Eje 3: "México con Educación de Calidad”. Revista Latinoamericana de Estudios Educativos, 43(2), 5-10. Recuperado de http:// www.redalyc.org/articulo.oa?id $=27028897001$

Vaillant, D. (Septiembre, 2007). La identidad docente. La importancia del profesorado. En I Congreso Internacional "Nuevas Tendencias en la Formación Permanente del Profesorado", Barcelona. Recuperado de http:// denisevaillant.com/articulos/2008/IdenDocFRONTERA2008.pdf

Van Dijk, T. A. (1999). Ideology. Londres: Sage

Vasilachis, I. (Coord.) (2006). Estrategias de Investigación cualitativa. Barcelona: Editorial Gedisa. Recuperado de: http://jbposgrado.org/icuali/investigacion\% 20cualitativa.pdf

Veiravé D., Ojeda, M., Núñez, C. y Delgado, P. (2006). La construcción de la identidad de los profesores de enseñanza media. Biografías de profesores. Revista Iberoamericana de Educación, 40(3), 1-11. Recuperado de https:// rieoei.org/RIE/issue/view/228

Zeichner, K. y Gore, J. (1990). Teacher socialization. En W. R. Houston (Ed.), Handbook of research on teacher education. New York: Macmillan

\section{BY-NC-ND}

